\title{
Quantum Ratchets with Few Bands below the Barrier
}

\author{
M. Grifoni, ${ }^{1}$ M. S. Ferreira, ${ }^{1,2}$ J. Peguiron, ${ }^{1}$ and J. B. Majer ${ }^{1}$ \\ ${ }^{1}$ Department of Nanoscience, Delft University of Technology, Lorentzweg 1, 2628 CJ Delft, The Netherlands \\ ${ }^{2}$ Department of Physics, University of Dublin, Trinity College, Dublin 2, Ireland
}

(Received 9 April 2002; published 11 September 2002)

\begin{abstract}
We investigate directed motion in nonadiabatically rocked ratchet systems sustaining few bands below the barrier. Upon restricting the dynamics to the lowest $M$ bands, the total system-plus-bath Hamiltonian is mapped onto a discrete tight-binding model containing all the information both on the intrawell and interwell tunneling motion. A closed form for the current in the incoherent tunneling regime is obtained. In effective single-band ratchets, no current rectification occurs. We apply our theory to describe rectification effects in vortex quantum ratchets devices. Current reversals upon variation of the ac-field amplitude or frequency are predicted.
\end{abstract}

DOI: 10.1103/PhysRevLett.89.146801

A ratchet, i.e., a periodic structure with broken spatial symmetry, yields the possibility to obtain a directed current in the presence of noise and unbiased nonequilibrium forces [1]. As such, ratchets belong to the class of so-termed Brownian motors [2], being devices operating far from equilibrium, and which combine noise and asymmetry to generate a particle's transport. A huge amount of experimental and theoretical work exists on ratchets ruled by classical thermal fluctuations [1,2], also due to their possible application in biological systems $[3,4]$. In contrast, little is known about the ratchet effect in the quantum realm. This is partly due to the challenge in the experimental realization of suitable ratchet potentials. Only recently has rectification of quantum fluctuations in triangularly shaped semiconductor heterostructures [5] and in quasi-one-dimensional Josephson junction arrays [6] been observed. Theoretically, this originates from the complexity in the investigation of the quantum ratchet dynamics. By now, the quantum ratchet effect has been tackled only for adiabatically rocked ratchets in a mostly numerical work [7], for peculiar single-band models [8], in the presence of an external force of on-off type [9], and for a weakly corrugated potential [10]. Typical quantum features, as, e.g., a current inversion upon temperature decrease, were predicted [7] and demonstrated [5]. So far, the band structure of the potential was not considered.

In this Letter for the first time a microscopic theory for the interplay among tunneling, vibrational relaxation, and nonadiabatic driving in ratchet potentials sustaining few bands below the barrier (cf. Fig. 1) is presented. For these potentials the semiclassical requirement [7] of having many bands below the barrier is not met. Our treatment, mostly analytical, is based on the real-time pathintegral formalism for open quantum systems [11]. Noticeably, in the temperature and driving regime in which the dynamics is effectively restricted to the lowest band of the periodic potential, no current rectification occurs. In fact, a reduction to the lowest band of the ratchet potential retains only information about the pe-
PACS numbers: 73.23.-b, 05.30.-d, 05.40.-a, 85.25.-j

riodicity of the original Hamiltonian, but not about its reflection properties. To take into account the vibrational motion within the well, and hence the asymmetry of the ratchet potential leading to the ratchet effect, at least two bands should contribute. Starting from the system-plusreservoir model of a driven ratchet system bilinearly coupled to a harmonic oscillator bath, we proceed in three steps: (i) We consider the regime of temperature and driving parameters which allow the truncation of the full dynamics to the Hilbert space spanned by the $M$ lowest bands of the periodic ratchet potential. (ii) A rotation to the so-called discrete variable representation (DVR) [12], being the eigenbasis of the discrete position operator coupling to the bath, is performed. In this basis, the isolated ratchet problem is described by a tightbinding model with a periodicity of $M$ sites, and with further nearest-neighbor coupling. (iii) The bath degrees of freedom are traced out exactly in the DVR basis, and a closed form expression for the averaged current in the incoherent tunneling regime is obtained. Subsequently, our results are applied to investigate current rectification and its optimization in ac-driven ratchets.

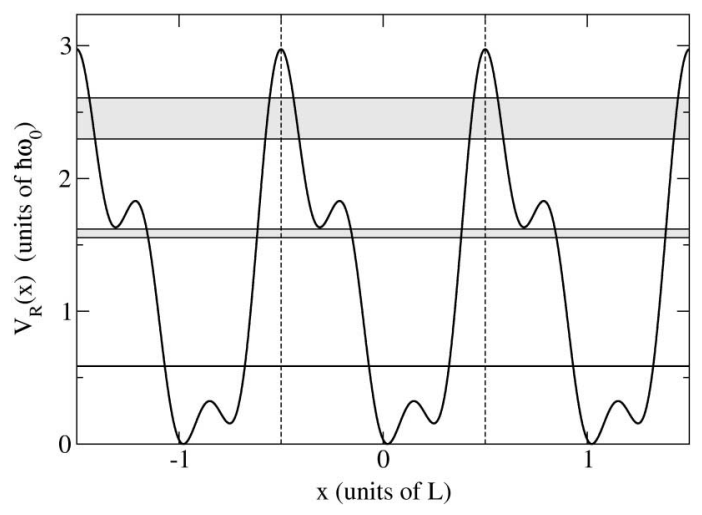

FIG. 1. Ratchet potential with three bands below the barrier (shaded regions). The potential height is in units of the distance $\hbar \omega_{0}$ between the centers of the second and first band, and the length is in units of the period $L$. 
We start considering the total Hamiltonian $H(t)=$ $H_{\mathrm{R}}+H_{\text {ext }}(t)+H_{\mathrm{B}}$, where $H_{\mathrm{R}}=p^{2} / 2 m+V_{\mathrm{R}}(x)$ is the Hamiltonian for a particle of mass $m$ moving in the asymmetric periodic ratchet potential $V_{\mathrm{R}}(x+L)=$ $V_{\mathrm{R}}(x)$; cf. Fig. 1. Because of the Bloch theorem, the spectrum of the isolated system follows from the Schrödinger equation $H_{\mathrm{R}}\left|\Psi_{n, k}\right\rangle=\mathcal{E}_{n}(k)\left|\Psi_{n, k}\right\rangle$, with $n$ denoting the band index and with $k$ being the wave vector. Time-reversal symmetry plus periodicity yield $\mathcal{E}_{n}(k)=$ $E_{n}+\sum_{m=1}^{\infty}\left(\Delta_{n}^{(m)} / 2\right) \cos (m k L)$. The action of the unbiased force is captured by $H_{\text {ext }}(t)=x F(t)$, with $F(t)=$ $F \cos \Omega t$, while $H_{\mathrm{B}}$ is the standard Hamiltonian for a harmonic oscillator bath with bilinear coupling in the bath and system coordinates [11]. The character of the bosonic bath is then fully described by the spectral function $J(\omega)$. Such a quantity is obtained from the equilibrium noise properties of the real system to be described. We assume the Ohmic form, relevant, e.g., in quasi-onedimensional Josephson junction arrays, $J(\omega)=\eta \omega /[1+$ $\left.\left(\omega / \omega_{\mathrm{D}}\right)^{2}\right]$, corresponding to a white noise spectrum in the classical limit, with $\eta$ being the friction coefficient and with $\omega_{\mathrm{D}}$ a bath cutoff frequency.

We introduce Wannier states localized in cell $j$ as $|n, j\rangle=(\sqrt{L / 2 \pi}) \int_{-\pi / L}^{\pi / L} d k e^{-i k j L}\left|\Psi_{n, k}\right\rangle$. Because of a lack of inversion symmetry, it holds $\langle x \mid j, n\rangle:=$ $\Psi_{j, n}(x) \neq \Psi_{-j, n}(-x)$ for some $x$. We restrict our attention to the lowest $M$ bands of the potential. We focus on Hamiltonians whose spectrum is well approximated by tight-binding Hamiltonians with only nearest-neighbor coupling; i.e., $\mathcal{E}_{n}(k)=E_{n}+\left(\Delta_{n} / 2\right) \cos k L$.

Then the ratchet Hamiltonian is fully characterized by the $2 M$ parameters, $\left\{\Delta_{n}, E_{n}\right\}, n=1, \ldots, M$, determining the bandwidths and the band centers, respectively. It has the form in the Wannier representation

$$
\begin{aligned}
H_{\mathrm{R}}= & \sum_{j=-\infty}^{\infty} \sum_{n=1}^{M} \frac{\Delta_{n}}{4}(|j, n\rangle\langle j+1, n|+| j+1, n\rangle\langle j, n|) \\
& +\sum_{j=-\infty}^{\infty} \sum_{n=1}^{M} E_{n}|j, n\rangle\langle j, n| .
\end{aligned}
$$

Correspondingly, the discrete position operator reads $x=$ $\sum_{j=-\infty}^{\infty} \sum_{n, m=1}^{M} x_{j}(n, m)|j, n\rangle\langle j, m|$, where, due to the localized character of the Wannier states, intercell matrix elements were neglected. Here, $x_{j}(n, m)=$ $\langle j, m|x| j, n\rangle:=j L \delta_{n m}+\xi_{n m}$, where the matrix $\xi_{n m}=$ $\xi_{m n}$ carries information on the shape of the ratchet potential $V_{\mathrm{R}}(x)$ within a cell. Only within the commonly performed lowest band truncation, $M=1$, is the position operator $x$ diagonal in the Wannier basis.

We wish to evaluate the average particle's velocity

$$
v=\left\langle v_{\text {as }}(t)\right\rangle_{\Omega}:=\left\langle\lim _{t \rightarrow \infty} \operatorname{Tr}\{x \dot{\rho}(t)\}\right\rangle_{\Omega}
$$

Here $\rho(t)=\operatorname{Tr}_{\text {Bath }} W(t)$ is the reduced density matrix of the system. It is obtained by performing the trace over the bath modes in the density matrix $W(t)$ of the system-plusreservoir. Finally, $\langle\cdots\rangle_{\Omega}$ denotes the time average over a driving period. The evaluation of the velocity $v$ is quite intricate: As clearly seen above, the coupling to the thermal bath and to the external field modifies the quantum coherent Bloch picture in (1). Being that the position operator is not diagonal in the Wannier basis, interband transitions become possible due both to the coupling to the thermal bath and to the external field.

For a harmonic bath bilinearly coupled to the system, the trace over the bath degrees of freedom can be done exactly by use of real-time path-integral techniques. This requires one, however, to express the full system-plusbath Hamiltonian $H(t)$ in the basis of the eigenstates of the position operator, the so-termed DVR $[12,13]$. The contributions $H_{\text {ext }}(t)$ and $H_{\mathrm{B}}$ to $H(t)$ are already diagonal in this basis, but not the isolated ratchet Hamiltonian. Diagonalization of the matrix $\xi_{n m}$ yields the orthogonal transformation $U$ from the Wannier to the DVR representation, $|j, n\rangle=\sum_{\mu} U_{n, \mu}|j, \mu\rangle$, as well as the position operator eigenvalues and eigenvectors $x|j, \mu\rangle=$ $x_{j, \mu}|j, \mu\rangle=\left(j L+x_{\mu}\right)|j, \mu\rangle$. Here $\mu$ labels the DVR states within a cell. Thus, the ratchet Hamiltonian reads in the DVR basis

$$
H_{\mathrm{R}}=\sum_{j=-\infty}^{\infty}\left(\sum_{\mu=1}^{M} \epsilon_{\mu}|j, \mu\rangle\left\langle j, \mu\left|+\sum_{\mu \neq \nu=1}^{M} \Delta_{\mu, \nu}^{\mathrm{intra}}\right| j, \mu\right\rangle\langle j, \nu|\right)+\sum_{j=-\infty}^{\infty} \sum_{\mu, \nu=1}^{M} \Delta_{\mu, \nu}^{\mathrm{inter}}(|j, \mu\rangle\langle j+1, \nu|+| j+1, \mu\rangle\langle j, \nu|),
$$

where the onsite energies read $\epsilon_{\mu} \equiv \sum_{n} E_{n} U_{n \mu} U_{n \mu}$, while $\Delta_{\mu, \nu}^{\mathrm{intra}} \equiv \sum_{n} E_{n} U_{n \mu} U_{n \nu}$ are related to the intracell vibrational motion. Finally, $\Delta_{\mu, \nu}^{\mathrm{inter}} \equiv \sum_{n}\left(\Delta_{n} / 4\right) U_{n \mu} U_{n \nu}$ accounts for intercell tunneling. Tunneling transitions to any DVR state in the neighboring cell are allowed. Additionally, because $H_{\text {ext }}(t)$ is diagonal in the DVR basis, the Hamiltonian $H(t)$ has time-dependent onsite energies $F(t) x_{j, \mu}$ which add to the intrinsic ones $\epsilon_{\mu}$. We have now all the ingredients to evaluate the asymptotic tunneling current. Its expression reads

$$
v_{\text {as }}(t)=\lim _{t \rightarrow \infty} \sum_{j=-\infty}^{\infty} \sum_{\mu=1}^{M} x_{j, \mu} \dot{P}_{j, \mu}(t)
$$

with $P_{j, \mu}(t)$ being the diagonal elements of the reduced density matrix in the DVR basis. They represent the probability of finding the particle in the state $|j, \mu\rangle$ at time $t$. In the following, we restrict our attention to the incoherent tunneling regime, reached at high enough temperatures and/or friction [11]. Moreover, we consider 
moderate-to-high frequencies $\Omega$, such that $\omega_{0} \geq \Omega>$ $\Gamma_{\nu, \mu}^{j, j^{\prime}}$, with $\Gamma_{\nu, \mu}^{j, j^{\prime}}$ being the field-averaged transition rate from DVR site $|j, \nu\rangle$ to $\left|j^{\prime}, \mu\right\rangle$. The upper bound, with $\hbar \omega_{0}=E_{2}-E_{1}$, is needed because of consistency with the truncation of the original problem to the first $M$ bands. The lower bound enables one to approximate the evolution of the averaged occupation probabilities $\bar{P}_{j, \mu}(t):=$ $\left\langle P_{j, \mu}(t)\right\rangle_{\Omega}$ by the coupled equations

$$
\begin{aligned}
\frac{d \bar{P}_{j, \mu}(t)}{d t}= & -\sum_{\nu \neq \mu}\left[\Gamma_{\mu, \nu}^{j, j} \bar{P}_{j, \mu}(t)-\Gamma_{\nu, \mu}^{j, j} \bar{P}_{j, \nu}(t)\right] \\
& -\sum_{\nu}\left[\Gamma_{\mu, \nu}^{j, j-1}+\Gamma_{\mu, \nu}^{j, j+1}\right] \bar{P}_{j, \mu}(t) \\
& +\sum_{\nu}^{\nu}\left[\Gamma_{\nu, \mu}^{j-1, j} \bar{P}_{j-1, \nu}(t)+\Gamma_{\nu, \mu}^{j+1, j} \bar{P}_{j+1, \nu}(t)\right] .
\end{aligned}
$$

Within an incoherent tunneling description, it is appropriate to evaluate such rates as an expansion in terms of the coupling matrix elements $\Delta_{\mu, \nu}^{j, j{ }^{\prime}}$, with $\Delta_{\mu, \nu}^{j, j}=\Delta_{\mu, \nu}^{\mathrm{intra}}$ and $\Delta_{\mu, \nu}^{j, j \pm 1}=\Delta_{\mu, \nu}^{\text {inter }}$. To lowest order, we find

$$
\begin{aligned}
\Gamma_{\mu, \nu}^{j, j j^{\prime}}= & \left(\Delta_{\mu, \nu}^{j, j^{\prime}} / \hbar\right)^{2} \int_{-\infty}^{\infty} d \tau e^{-Q_{\mu, \nu}^{j, j^{\prime}}(\tau)} \\
& \times J_{0}\left[\frac{2 F\left(x_{j, \mu}-x_{j^{\prime}, \nu}\right)}{\hbar \Omega} \sin \left(\frac{\Omega \tau}{2}\right)\right] e^{i\left(\epsilon_{\mu}-\epsilon_{\nu}\right) \tau / \hbar},
\end{aligned}
$$

where the external field parameters enter the argument of the zero-order Bessel function $J_{0}$. This yields a strong reduction of the rate (5), when $\epsilon_{\mu}-\epsilon_{\nu}=n \hbar \Omega$ and the ratio $F\left(x_{j, \mu}-x_{j^{\prime}, \nu}\right) / \hbar \Omega$ hits a zero of the Bessel function $J_{n}$. In the absence of dissipation, similar conditions would yield a dynamical-localization of the particle [14,15]. However, finite temperatures of the bath hinder this effect. The environmental influence is determined by the bath correlation function $Q_{\mu, \nu}^{j, j^{\prime}}(\tau)=\frac{\left(x_{j, \mu}-x_{j^{\prime}, \nu}\right)^{2}}{2 \pi \hbar} \int_{0}^{\infty} d \omega \frac{J(\omega)}{\omega^{2}}[\operatorname{coth}(\hbar \beta \omega / 2)(1-$ $\cos \omega \tau)+i \sin \omega \tau]$ [11]. Note that the dissipation strength depends on the square distance between the DVR states involved. It is position dependent through the dimensionless coupling parameter

$$
\alpha_{\mu, \nu}^{j, j^{\prime}}=\frac{\eta}{2 \pi \hbar}\left(x_{j, \mu}-x_{j^{\prime}, \nu}\right)^{2} .
$$

For symmetric cosine shaped potentials, a bath-induced localization at zero temperatures has been predicted for dissipation parameters $\alpha=\eta L^{2} /(2 \pi \hbar)>1$ [16]. A similar tendency to localization occurs in our ratchet for large dissipation strengths $\alpha_{\mu, \nu}^{j, j \pm 1}>1$ and zero temperature. However, appropriately tuned ac fields can help prevent localization.

The stationary solutions of the coupled system of Eqs. (4) can be found upon Laplace transformation. Then, the current assumes the compact form

$$
v=L \sum_{\nu, \mu} p_{\nu}^{\infty}\left(\Gamma_{\nu, \mu}^{\mathrm{inter}, f}-\Gamma_{\nu, \mu}^{\mathrm{inter}, b}\right),
$$

where we introduced the forward and backward timeaveraged rates $\Gamma_{\mu, \nu}^{\mathrm{inter}, f / b}:=\Gamma_{\mu, \nu}^{j, j \pm 1}$ and the vibrational relaxation rates $\Gamma_{\mu, \nu}^{\text {intra }}:=\Gamma_{\mu, \nu}^{j, j}(\mu \neq \nu)$. The asymptotic cell occupation probabilities $p_{\mu}^{\infty}:=\lim _{t \rightarrow \infty} \sum_{j} \bar{P}_{j, \mu}(t)$ are expressed in terms of the averaged rates. For $M=1$, the current in (7) is zero, as a consequence of time-reversal symmetry. Thus single-band quantum ratchets support no current, as recently reported in [6]. They define a new class of supersymmetric [1] potentials in the quantum regime. At least two bands should contribute to the dynamics in order to have a nonvanishing current. For $M=$ 3 contributing bands (or DVR states), we find

$$
p_{1}^{\infty}=\frac{\Gamma_{2} \Gamma_{3}-\Gamma_{2,3} \Gamma_{3,2}}{\sum_{\nu=1}^{3} \sum_{\gamma>\nu=1}^{3}\left(\Gamma_{\nu} \Gamma_{\gamma}-\Gamma_{\nu, \gamma} \Gamma_{\gamma, \nu}\right)},
$$

where $\Gamma_{\nu}=\sum_{\mu \neq \nu} \Gamma_{\nu, \mu}$ and $\Gamma_{\nu, \mu}:=\Gamma_{\nu, \mu}^{\text {inter, } f}+\Gamma_{\nu, \mu}^{\text {inter, } b}+$ $\Gamma_{\nu, \mu}^{\text {intra }}$. Likewise, $p_{2}^{\infty}$ and $p_{3}^{\infty}$ can be obtained from (8) upon cyclic index permutation. In the absence of ac driving, the rates (5) obey the detailed-balance relation

$$
\Gamma_{\mu, \nu}^{j, j^{\prime}}=\Gamma_{\nu, \mu}^{j^{\prime}, j} e^{-\left(\epsilon_{\mu}-\epsilon_{\nu}\right) / k_{\mathrm{B}} T},
$$

so that the total current in (7) adds up to zero. The ac field invalidates (9), and a net current is originated.

Up to this point, our results are general. We apply now our theory to make predictions for vortex motion in quantum ratchets devices. These could be realized with rectangular [6] or circular [17] arrays of different-sized Josephson junctions. Upon applying a magnetic field perpendicular to the array, current vortices are originated, whose dynamics is homologous to that of a quantum Brownian particle in a periodic ratchet potential $[18,19]$. The particle's mass is $m=\Phi_{0}^{2} C / 2 a^{2}$, where $\Phi_{0}$ is the flux quantum, $C$ is the average capacitance of the junctions, and $a$ is the average junction distance in the direction of motion. The magnitude of the potential felt by the vortices is proportional to the Josephson energy $E_{J}$. The vortices can be put into motion upon injecting a current $I=I_{\mathrm{AC}} \cos (\Omega t)$ into the array, which exerts a Lorentzlike force on such particles. At low vortex densities, the voltage drop $V$ across the array is related to the vortex velocity by the second Josephson relation,

$$
V=s v \Phi_{0} / L,
$$

where $s$ is the one-dimensional vortex density. Figures 2 and 3 illustrate our predictions for current rectification for the driven ratchet potential shown in Fig. 1. It supports only three bands below the barrier, being characterized by $\Delta_{1}=-4.43 \times 10^{-3} \hbar \omega_{0}, \Delta_{2}=$ $6.68 \times 10^{-2} \hbar \omega_{0}$, and $\Delta_{3}=-3.10 \times 10^{-1} \hbar \omega_{0}$. The center of the bands read $E_{1}=5.87 \times 10^{-1} \hbar \omega_{0}$, $E_{2}=1.59 \hbar \omega_{0}$, and $E_{3}=2.45 \hbar \omega_{0}$. This is the potential reported in [6] at a temperature of $T=50 \mathrm{mK}$, corresponding to a charging energy $E_{J}=429 \mu \mathrm{eV}=$ $8 \hbar \omega_{0}$. We choose an average capacitance $C=2 \mathrm{fF}$, an average distance $a=L / 3=2 \mu \mathrm{m}$, and frustration $s=$ 0.277 . The average junction resistance $R$ is taken as a free parameter. It determines the friction parameter $\eta \approx \frac{\Phi_{0}^{2}}{2 R a^{2}}$ and the bath cutoff $\omega_{\mathrm{D}} \approx(R C)^{-1}$ [19]. In Figs. 2 and 3 we 


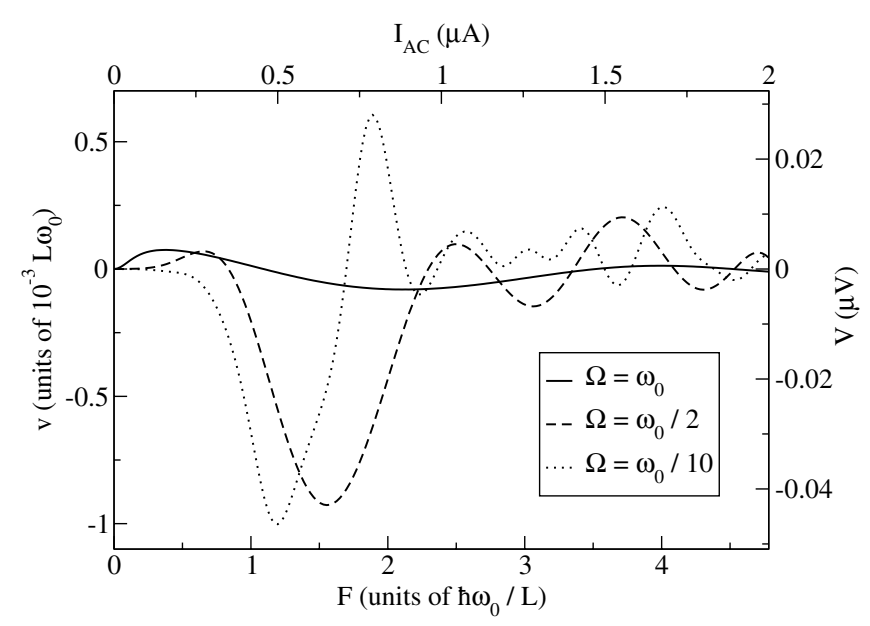

FIG. 2. Output velocity (or dc voltage for the vortex ratchet case) vs amplitude of the driving ac signal (ac current for vortex ratchet) for different driving frequencies $\Omega$. The dimensionless friction parameter $\alpha=2 \pi \eta L^{2} / \hbar$ is chosen to be $\alpha=$ 0.5 , describing moderate damping. Negative or positive ratchet currents are obtained upon appropriate tuning of the driving field parameters.

focus on two different values of the dimensionless friction parameter $\alpha$, cf. below (6), i.e., $\alpha=0.5$ and $\alpha=2.0$ which describe a moderate and a strong damping regime, respectively. These fully specify the ratchet potential and the bath parameters. Finally, the injected ac current induces a force $F(t)=I_{\mathrm{AC}} \cos (\Omega t) \Phi_{0} / N_{c} a$, where $N_{c}=$ 304 is the chosen number of junction columns of the array. Figures 2 and 3 reveal how the interplay among driving-induced localization near the zeros of the Bessel function in (5), driving-assisted tunneling when the driving frequency matches resonances of the dissipative ratchet system, and dissipation results in a nontrivial nonmonotonic dependence of the current on the applied ac amplitude and frequency. Likewise, a nonmonotonic behavior occurs, varying damping. For example, different current directions are observed for small ac amplitudes in the curves with driving $\Omega=\omega_{0}$ of Figs. 2 and 3. This inversion also occurs in combination with a change in the bath cutoff frequency $\omega_{\mathrm{D}}$. As damping is further increased, the main resonance peaks get shifted to even larger values of the driving amplitude, and the ratchet signal is reduced.

In conclusion, we investigated the ratchet mechanism in periodic quantum structures with few bands below the barrier, and driven by nonadiabatic time-dependent fields. A set of coupled master equations for the reduced density matrix elements in the basis which diagonalizes the position operator are derived. The validity of our method is restricted to the deep quantum regime, since thermally activated processes over the barrier are disregarded. Our approach is complementary to the semiclassical analysis of [7], where many bands below the barrier are required. We discussed current rectification in

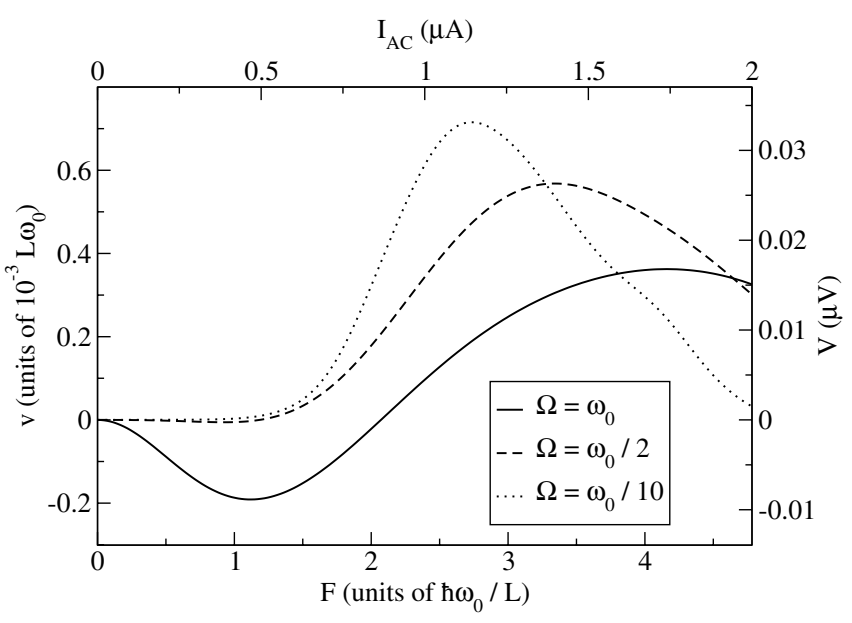

FIG. 3. Same as in Fig. 2 for larger friction, $\alpha=2.0$. The main resonances occur at larger driving amplitudes.

Josephson ratchets. Current reversals result from the interplay of driving-induced dynamical localization [14] versus dissipation-assisted diffusion.

We thank M. Thorwart, P. Hänggi, and J. E. Mooij for useful discussions. Support by FOM is acknowledged.

[1] P. Reimann, Phys. Rep. 361, 57 (2002).

[2] Special issue on Ratchets and Brownian motors: Basics, experiments and applications [Appl. Phys. A 75 (2002)].

[3] J. Maddox, Nature (London) 365, 203 (1993); 365, 181 (1994).

[4] R. D. Astumian, Science 276, 917 (1997).

[5] H. Linke et al., Science 286, 2314 (1999).

[6] J. B. Majer et al. (to be published).

[7] P. Reimann, M. Grifoni, and P. Hänggi, Phys. Rev. Lett. 79, 10 (1997).

[8] R. Roncaglia and G. P. Tsironis, Phys. Rev. Lett. 81, 10 (1998).

[9] S. Yukawa, M. Kikuchi, G. Tatara, and H. Matsukawa, J. Phys. Soc. Jpn. 66, 2953 (1997).

[10] S. Scheidl and V. M. Vinokur, Phys. Rev. B 65, 195305 (2002).

[11] U. Weiss, Quantum Dissipative Systems (World Scientific, Singapore, 1999), 2nd ed.

[12] D. O. Harris, G. G. Engerholm, and W. D. Gwinn, J. Chem. Phys. 43, 1515 (1965).

[13] M. Thorwart, M. Grifoni, and P. Hänggi, Phys. Rev. Lett. 85, 860 (2000).

[14] M. Grifoni and P. Hänggi, Phys. Rep. 304, 229 (1998).

[15] D. H. Dunlap and V. M. Kenkre, Phys. Rev. B 34, 3625 (1986).

[16] A. J. Bray and M. A. Moore, Phys. Rev. Lett. 49, 1545 (1982).

[17] F. Falo et al., Appl. Phys. A 75, 263 (2002).

[18] C. J. Lobb, D. W. Abraham, and M. Tinkham, Phys. Rev. B 27, 150 (1983).

[19] T. P. Orlando, J. E. Mooij, and H. S. J. van der Zant, Phys. Rev. B 43, 10218 (1991). 